\title{
Broadband coherent perfect absorption in epsilon- near-zero bilayer planar thin film
}

\author{
Md. Alamgir Badsha* \\ Department of Physics, Jashore University of Science and Technology, Jashore-7408, Bangladesh. \\ ${ }^{(*)}$ E-mail:alamgir93_phy@just.edu.bd \\ Received: 30/10/2020 Accepted: 06/02/2021 \\ DOI: 10.7149/OPA.54.1.51053
}

\begin{abstract}
Interactions of electromagnetic wave with epsilon-near-zero (ENZ) bilayer planar thin films have been extracted in near-infrared. The ENZ material properties are harnessed theoretically as broadband and narrowband coherent perfect absorption (CPA). A dielectric layer is deposited with a tunable ITO layer on substrate for analysis of narrowband CPA. The broadband spectral regimes of CPA are produced at bilayer of tunable ITOs. The designs of these planar thin films are carried out by admittance matching method at the illumination of two counter input beams. The CPA condition is obtained by this method and the mode is characterized in effective medium approximation (EMA). It develops the eigenvalue expression of CPA mode that behaves like the transverse resonance phase matching status. The output irradiance expressions are established with phase controlling beams in scattering matrix method. These devices are applicable in energy conversion, optical switching, modulation and infrared sensor at the communication system.
\end{abstract}

Keywords: Absorption, Interference, Sensor, Modulation and Integrated optics.

\section{REFERENCES AND LINKS:}

[1] Simin Feng, Klaus Halterman, "Coherent perfect absorption in epsilon-near-zero metamaterials", Phys. Re. B, 86, 165103 (2012).

[2] Andrea Alù, Nader Engheta, "Dielectric sensing in $\varepsilon$-near-zero narrow waveguide channels", Phys. Re. B, 78, 045102 (2008).

[3] Jie Luo, Ping Xu, Lei Gao, Yun Lai, Huanyang Chen, "Manipulate the transmissions using index-near-zero or epsilon-near-zero metamaterials with coated defects", Plasmonics, 7, 353-358 (2012).

[4] Young Chul Jun, John Reno, Troy Ribaudo, Eric Shaner, Jean-Jacques Greffet, Simon Vassant, Francois Marquier, Mike Sinclair, Igal Brener, "Epsilon-near-zero strong coupling in metamaterial-semiconductor hybrid structures", Nano lett., 13, 5391-5396 (2013).

[5] Shuomin Zhong, Yungui Ma, Sailing He, "Perfect absorption in ultrathin anisotropic e-near-zero metamaterials", App. Phy. Let., 105, 023504 (2014).

[6] Klaus Halterman, J. Merle Elson, "Near-perfect absorption in epsilon-near-zero structures with hyperbolic dispersion", Opt. Exp., 22, 7337-7348 (2014).

[7] Nadav Gutman, Andrey A. Sukhorukov, Y. D. Chong, C. Martijn de Sterke, "Coherent perfect absorption and reflection in slow-light waveguides", Opt. Let., 38, 4970-4973 (2013).

[] Heeso Noh, Yidong Chong, A. Douglas Stone, Hui Cao, "Perfect coupling of light to surface plasmons by coherent absorption", PRL, 108, 186805 (2012).

[9] Alamgir Badsha, "Coherent perfect absorption in plasmonic planar metasurface", Int. J. of Physical Sciences, 15, 213-220 (2020).

[10] Y. D. Chong, Li Ge, Hui Cao, A. D. Stone, Coherent perfect absorbers: time-reversed lasers, PRL, 105, 053901 (2010). 
[11] Wenjie Wan, Yidong Chong, Li Ge, Heeso Noh, A. Douglas Stone, Hui Cao, "Time-reversed lasing and interferometric control of absorption”, SCIENCE, 331, 889-892 (2011).

[12] Md. Alamgir Badsha, Young Chul Jun, Chang Kwon Hwangbo, "Admittance matching analysis of perfect absorption in unpatterned thin films", Opt. Comm. 332, 206-213 (2014).

[13] Junho Yoon, Md. Alamgir Badsha, Tae Young Kim, Young Chul Jun, Chang Kwon Hwangbo, "Tunable and Broadband Perfect Absorption in Epsilon-Near-Zero Indium Tin Oxide Thin Films at Near Infrared Wavelengths, Conference on Lasers and Electro-Optics Pacific Rim. paper 26I2_2, (Optical Society of America, 2015).

[14] Ming Kang, Fu Liu, Teng-Fei Li, Qing-Hua Guo, Jensen Li, Jing Chen, "Polarization-independent coherent perfect absorption by a dipole-like metasurface", Opt. Let., 38, 3086-3088 (2013).

[15] Shourya Dutta-Gupta, O. J. F. Martin, S. Dutta Gupta, G. S. Agarwal, "Controllable coherent perfect absorption in a composite film", Opt. Exp., 20, 1330-1336 (2012).

[16] Md. Alamgir Badsha, Mohammad Abdur Rashid, Md. Humaun Kabir, Md. Mehade Hasan, "Coherent perfect absorption in epsilon-near-zero ITO thin film in near infrared", Opt. Pura Apl., 53, 1-12 (2020).

[17] Tae Young Kim, Md. Alamgir Badsha, Junho Yoon, Young Chul Jun, Chang Kwon Hwangbo, "Design of epsilon-near-zero coherent perfect absorption with indium tin oxide thin films using admittance matching method", Conference on Lasers and Electro-Optics Pacific Rim, paper 27P_62, (Optical Society of America, 2015).

[18] Wangyang Li, Yongzhi Cheng, "Dual-band tunable terahertz perfect metamaterial absorber based on strontium titanate (STO) resonator structure", Opt. Comm., 462, 125265 (2020).

[19] Feng Qin, Zeqiang Chen, Xifang Chen, Zao Yi, Weitang Yao, Tao Duan, Pinghui Wu, Hua Yang, Gongfa Li, Yougen Yi, "Tunable triple-band near-infrared metamaterial absorber based on Au nano-cuboids array", Nanomaterials, 10, 207 (2020).

[20] Fu Chen, Yongzhi Cheng, Hui Luo, "A broadband tunable terahertz metamaterial absorber based on singlelayer complementary Gammadion-shaped Graphene", Materials, 13, 860 (2020).

[21] Yongzhi Cheng, Hui Luo, Fu Chen, "Broadband metamaterial microwave absorber based on asymmetric sectional resonator structures", J. Appl. Phys., 127, 214902 (2020).

[22] Mingbo Pu, Qin Feng, Min Wang, Chenggang Hu, Cheng Huang, Xiaoliang Ma, Zeyu Zhao, Changtao Wang, Xiangang Luo, "Ultrathin broadband nearly perfect absorber with symmetrical coherent illumination", Opt. Exp., 20, 2246 (2012).

[23] Jianfa Zhang, Chucai Guo, Ken Liu, Zhihong Zhu, Weimin Ye, Xiaodong Yuan, Shiqiao Qin, "Coherent perfect absorption and transparency in a nanostructured graphene film”, Opt. Exp., 22, 12524 (2014).

[24] Iñigo Liberal, Ahmed M. Mahmoud, Yue Li, Brian Edwards, Nader Engheta, "Photonic doping of epsilon-nearzero media", Science, 355, 1058-1062 (2017).

[25] Jie Luo, Bingbing Liu, Zhi Hong Hang, Yun Lai, "Coherent perfect absorption via photonic doping of zeroindex media, Laser Photonics Rev., 12, 1800001 (2018).

[26] Marino Coppolaro, Massimo Moccia, Giuseppe Castaldi, Nader Engheta, Vincenzo Galdi, "Non-Hermitian doping of epsilon-near-zero media", PNAS, 117, 13921-13928 (2020).

[27] Junho Yoon, Ming Zhou, Md. Alamgir Badsha, Tae Young Kim, Young Chul Jun, Chang Kwon Hwangbo, "Broadband epsilon-near-zero perfect absorption in the near-infrared", Scientific Reports 5, 12788 (2015).

[28] David J. Bergman, "The dielectric constant of a composite material-a problem in classical Physics", Physics Reports (Section of C of Physics Letters), 43, 377-407 (1978).

[29] L. Sun, K. W. Yu, "Strategy for designing broadband epsilon-near-zero metamaterials", J. Opt. Soc. Am. B, 29, 984-989 (2012).

[30] Angus Macleod, "Optical absorption part 2, Society of vacuum coaters", Bulletin 13, 28-33 (2013).

[31] Michal Lipson, "Guiding, modulating, and emitting light on silicon-challenges and Opportunities", J. L. W. Tech., 23, 4222-4238 (2005).

[32] Gururaj V. Naik, Vladimir M. Shalaev, Alexandra Boltasseva, "Alternative plasmonic materials: beyond gold and silver", Adv. Mater., 25, 3264-3294 (2013).

[33] Alok P. Vasudev, Ju-Hyung Kang, Junghyun Park, Xiaoge Liu, Mark L. Brongersma, "Electro-optical modulation of a silicon waveguide with an "epsilon-near-zero" material", Opt. Exp., 21, 26387-26396 (2013).

[34] Eyal Feigenbaum, Kenneth Diest, Harry A. Atwater, "Unity-order index change in transparent conducting oxides at visible frequencies", Nano Lett.,10, 2111-2116 (2010).

[35] Ho W. Lee, Georgia Papadakis, Stanley P. Burgos, Krishnan Chander, Arian Kriesch, Ragip Pala, Ulf Peschel, Harry A. Atwater, "Nanoscale conducting oxide plasMOStor", Nano Lett., 14, 6463-6468 (2014).

[36] H. A. Macleod. Thin-Film Optical Filters, $4^{\text {th }}$ edition, London, Taylor \& Francis, 2010. 
[37] Tae Young Kim, Md. Alamgir Badsha, Junho Yoon, Seon Young Lee, Young Chul Jun, Chang Kwon Hwangbo, "General strategy for broadband coherent perfect absorption and multi-wavelength all-optical switching based on epsilon-near-zero multilayer films", Scientific Reports, 6, 22941 (2016).

[38] Md. Alamgir Badsha, Md. Humaun Kabir and Mohammad Abdur Rashid, "Coherent perfect absorption in unpatterned thin films of intrinsic semiconductor", J. Opt., 49, 342-350 (2020).

[39] The dielectric constant of $\mathrm{SiO}_{2}$, https://refractiveindex.info/?shelf=main\&book=SiO2\&page=Malitson

[40] Essential Macleod thin film software package: 〈http://www.thinfilmcenter.com/essential.php〉.

\section{Introduction}

Nowadays manipulation of electromagnetic wave through interaction with ENZ material is being likely an upcoming technological trend. The potential application of photonic devices in the integrated optics is appreciable in this regard. Either loss or gain effect of light in the matter is a very acceptable phenomenon of optical sensing application. To make it practical, the different nanostructures of nanoparticle, grating and metamaterials (MMs) have been tailored and exploited in theoretical and experimental views. The 3D MMs are harvested promisingly for different applications. CPA is striking research topic for its various technological purposes through nanostructured MMs and recently in reconfigurable MMs as well.

The nanostructured ENZ MMs are being used in study of absorbing effect at low loss regime [1], field enhancement at near-zero effective permittivity [2] and matching electric permittivity enhanced transmittance is also achieved [3]. Controlling ENZ material doping densities in multilayered MM can produce chip scale active IR devices [4]. The anisotropic ENZ MM is verified to be controllable absorber and emitter by tuning the physical thickness and material loss effect [5]. The hyperbolic MM produces near-perfect absorption while the parallel component of permittivity is opposite in sign of normal component of permittivity [6]. Producing interference of propagating slow-light modes, total absorption appears also in infinite pattern of lossy waveguide [7]. Surface plasmon polariton assisted CPA is available in dielectric coated on metallic nanoparticle [8]. The energy dissipated medium of resonator [9] is very likely selective for appearance of complete absorption monochromatically [10, 11]. Critical coupling condition is usually attributable on CPA for single input beam $[12,13]$ and sometime for double input beam as well [14]. In mixed structure such as non-absorbing and absorbing layers generates monochromatic perfect absorption which is observable from absolute value of reflection and transmission coefficients [15-17]. Multiband near perfect absorber can be dependent on surrounding temperature and the structural parameters $[18,19]$. Electrically tunable broadband absorption is performed in graphene MM [20] as well.

The polarization insensitive broadband MM microwave absorber potentiality in stealth technology and energy harvesting system is not unlikely [21]. Heavily doped semiconductor causes broadband perfect absorption and the nanostructure of graphene thin film that yields also the CPA at terahertz frequency [22, 23]. The reconfigurable MM by photonic doping is the latest development to the researchers. This mechanism transforms the ENZ medium to the zero index medium. It can also produce CPA in multichannel illumination [24, 25]. Observation of dissipation and amplification of electromagnetic wave either in the dopant or in the ENZ host media and these surfaces bear another novel development of non-hermitian optics for optical sensing application [26].

From the above discussion it is noticeable that the study of CPA in ENZ material is the most promising and focusing domain for the researchers. The optical phenomenon which appears in the planar thin film [12,27] then it is more convenient to extract it there rather than the bulk nanostructured MMs and the reconfigurable MMs. In this consideration, the planar thin film of tunable ITO is used here to analyze the narrowband spectrum regime and broadband spectral regimes of $1498 \mathrm{~nm}-1641 \mathrm{~nm}$ as ENZ CPA. These CPAs are carried out in bilayer thin films. EMA $[28,29]$ is exploited to characterize the CPA modes. The admittance matching method provides the design and produces the transverse resonance like behavior of CPA mode in thin film structure. The two identical incident medium of $\mathrm{ZnSe}$ is used at illumination of two counter input beams in these designs for p-polarized light. The amplitude and phase matching conditions of CPA are also established here in scattering matrix method. These devices are applicable in sensor, detector [8], optical switching, modulator, energy conversion [10, 11], counter inputs absorber [30] and in the integrated optics [31]. 


\section{Analysis of dielectric constants}

\section{2. a. Dielectric constant of tunable ITO}

It is not convenient to adjust the electro-optical properties in the noble plasmonic materials for optoelectronics devices. Thus this difficulty leads to delve the finding of alternative plasmonic materials. The transparent conducting oxides (TCOs) are comparatively handy to achieve it. The elemental compositions of TCOs are defective in lattice structure and their electronic properties ensure the novel windows to apply these materials in the nanostructure MM and integrated electro-optical devices. In these regards, the growth technique and deposition condition are crucial for fabrication of TCO thin film [27, 32]. ITO is very much selective TCO for adjustable properties by electro-dynamical process in near-infrared (NIR) range. Inducing fractional real value of permittivity to longer wavelength and then the modulated ENZ position produces high absorption contrast value and enhances the coupling resonance at a suitable spectral regime of tunable ITO [4, 33]. In metal-oxide-semiconductor heterostructure, ENZ ITO plays a key role as active alternative plasmonic material with variation of complex dielectric function for nanoscale modulator [34,35]. As a dynamical field effect active material ITO is one of the chosen TCO. The role of NIR ENZ spectrum of this material is positively applicable for induction of opto-electrical properties. Thus the surface properties of ITO allure to employ it further investigation.

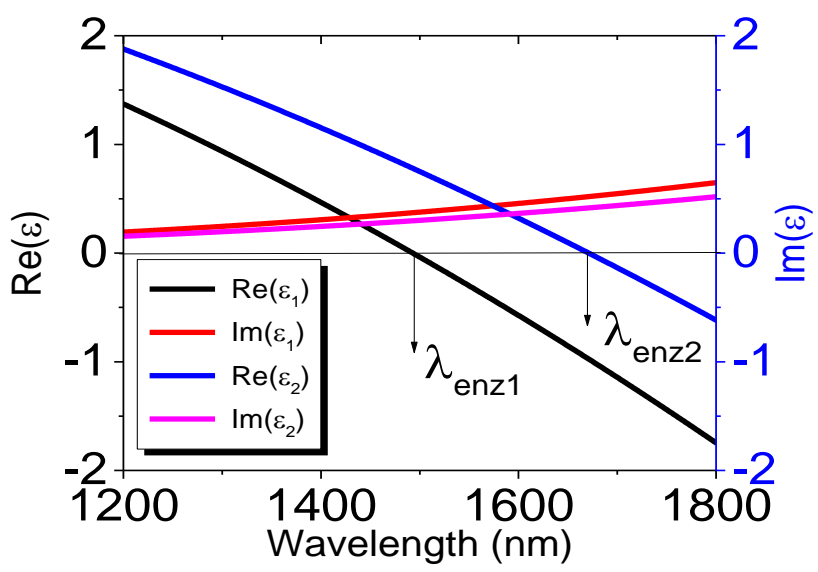

Figure 1: The dielectric constant $\varepsilon=(n-j k)^{2}$ of tunable ITO-1 and ITO-2 are drawn for both real and imaginary parts in wavelength. The real parts of dielectric constants are zero $\operatorname{Re}(\varepsilon)=0$ at the epsilon-near-zero (ENZ) wavelengths of $N_{\text {ITO-1 }}\left(1492 \mathrm{~nm}=\lambda_{\text {enz1 }}\right)=0.43335-0.427752 * 1 j$ and $N_{\text {ITO-2 }}\left(1671 \mathrm{~nm}=\lambda_{\text {enz2 }}\right)=$ $0.456023-0.45579 * 1 j$ respectively.

In this article, Drude model approximation is dealt on time dependent external electric field and position of the electron relative to the atom of harmonic $e^{-i \omega t}$ function. The optical analysis is done by that approximation [12] as

$$
\varepsilon(\omega)=(n-j k)^{2}=\varepsilon_{b}-\frac{\omega_{p}^{2}}{\omega^{2}\left(1+j \frac{\gamma}{\omega}\right)}
$$

where $n$ is the real part and $k$ is imaginary part of complex refractive index respectively. The $k$ is termed as extinction coefficient of the material. The background dielectric constant at infinite frequency is defined as $\varepsilon_{b}$, the plasma frequency $\omega_{p}=\sqrt{\frac{N_{0} e^{2}}{\varepsilon_{0} m_{e}}}$, where $N_{0}$ is the carrier density, $\varepsilon_{0}$ electric permittivity in the vacuum, $m_{e}$ effective mass of electron and $e$ is the electronic charge. Plasma frequency is also related to ENZ frequency $\omega_{e}$, damping frequency $\gamma$ and with the $\varepsilon_{b}$ such as $\omega_{p}=\sqrt{\varepsilon_{b}\left(\omega_{e}^{2}+\gamma^{2}\right)}$. The variation of the carrier concentration is dependent on doping densities, growth technique and tuning the external potential difference in the integrated active material $[4,33-35]$ that makes the shifting of the spectral position of ENZ and plasma frequency of ITO. The dispersion relation of Fig. 1 shows that around the cross-over frequency real parts and imaginary parts of dielectric constants 
are smaller than unity in values. These novel material properties are necessary for study of angle dependent of directional absorber especially in NIR regime in the material of ENZ ITO.

\section{2. b. Effective dielectric constant}

The effective medium approximation (EMA) is to apply for the analysis of electro-optical properties at the inhomogeneous layers. The sequential deposition of parallel layers to the substrate of different materials have always prerequisite of this approximation. The effective permittivity of the deposited multilayer of parallel planar thin films can be simplified $[28,29]$ as

$$
\varepsilon_{e f f}=\left[\sum_{i=1}^{l} \frac{f_{i}}{\varepsilon_{i}}\right]^{-1}
$$

where $\sum_{i=1}^{l} f_{i}=1$, is the total physical thickness of the multilayer thin film, $f_{i} \ll 1$, and $f_{i}$ is the fractional physical thickness of the individual layer which is calculated by ratio of specific layer thickness to the total layer thickness. The individual layer dielectric constant is represented by $\varepsilon_{i}$. Using the simplified eq. (2), the optical properties of multilayer can be calculated as single layer of EMA.

\section{Analysis of CPA Theory}

\section{3. a. Admittance matching method}

Doping nanostructure and reconfigurable MMs [22-25] have a curious mindset recently to the researchers due to getting some novel properties from these artificial surfaces. In spite of these, there is requirement of simple design in thin film arena. The Admittance matching method is that tool to anticipate planar thin film geometry before fabrication.

(a)

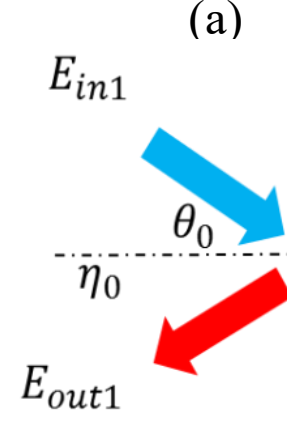

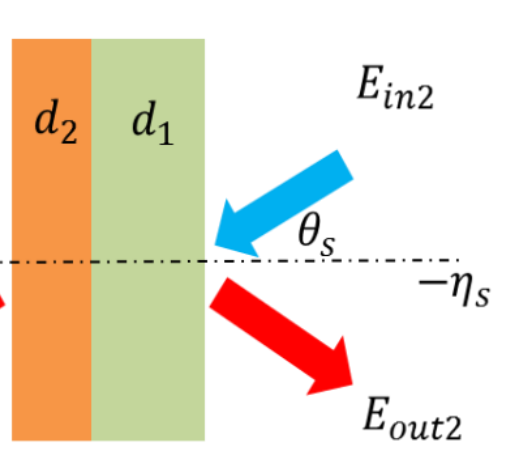

(b)
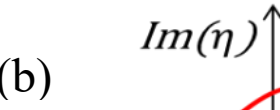

Figure 2: (a) The schematic diagram is drawn for a bilayer planar thin film in two counter input beams of ppolarized light from non-identical incident media and (b) The schematic admittance matching design is shown for that geometry of (a).

The design is completed by the transfer matrix method (TMM) as the eq. (3). The illustrated Fig. 2 (a) is used schematically. The modified admittance of incident and substrate medium is denoted by $\eta_{0}$ and $\eta_{s}$ respectively. The negative value of substrate admittance $\left(-\eta_{s}\right)$ is assigned due to the reversibility of Poynting vector in time reversal symmetry of illumination. The bilayer individual thickness is denoted by $d_{1}$ and $d_{2}$. The both layers can be deposited by absorbing medium with complex refractive index $N=n-j k$. The plasmonic and dielectric layers can also be deposited consecutively. The amplitudes of forward and backward input beams are indicated as $E_{\text {in } 1}$ and $E_{i n 2}$ respectively. The input beams are directed to the bilayer film from the incident medium at $\theta_{0}$ and from the substrate medium at $\theta_{s}$ respectively. Similarly the amplitudes of counter output emergent beams are shown as $E_{\text {out } 1}$ and $E_{\text {out } 2}$. 
The Fig. 2 (b) is sketched for presentation of the trajectories of admittance diagrams in a complex plane of modified admittance. The admittance diagrams of each beam start from its emergent medium. The diagram which launches at the coordinate of substrate medium and it is attributable to the forward input beam. It traverses in clockwise direction through the layer-1 which is denoted as $d_{1}$ (red color). The admittance diagram which starts at incident medium, it appears due to the backward input beam. It passes in anticlockwise direction through the layer-2 and it is symbolized as $d_{2}$ (blue color). The starting coordinates of each diagram is real such as $\left(-\eta_{s}, 0\right)$ and $\left(\eta_{0}, 0\right)$ respectively. Thus two diagrams travel in counter direction and cross at a certain complex coordinate of modified admittance. This crossing takes place when the surface admittance $\left(Y=\frac{C}{B}\right)$ of thin film geometry [36] is equal to the admittance of incident medium $\left(\eta_{0}\right)$. It ensures the satisfaction of CPA design. Using this method, the different required parameters of thin film geometry are easily achievable.

The symmetric geometry $\eta_{0}=\eta_{s}$ whereas the incident and substrate medium are optically identical that is utilized here for analysis of CPA. The EMA is exploited for extraction of optical properties in the bilayer thin film of planar geometry. In this regard, the transfer matrix can be written as

$$
\left[\begin{array}{l}
B \\
C
\end{array}\right]=\left[\begin{array}{cc}
\cos \delta_{e f f} & j \frac{\sin \delta_{e f f}}{\eta_{e f f}} \\
j \eta_{e f f} \sin \delta_{e f f} & \cos \delta_{e f f}
\end{array}\right]\left[\begin{array}{c}
1 \\
-\eta_{s}
\end{array}\right]
$$

where $B$ and $C$ are the normalized electric field and magnetic field respectively. The modified admittance of respective medium for p-polarized light is defined as $\eta_{0}=\frac{n_{0} \cos \theta_{0}}{\cos \theta_{0}}=n_{0}, \eta_{s}=\frac{n_{s} \cos \theta_{0}}{\cos \theta_{s}}, \quad \eta_{e f f}=\frac{N_{e f f} \cos \theta_{0}}{\cos \theta}$ for the incident, substrate and for the effective medium of bilayer respectively. Here $\theta_{0}$ and $\theta_{s}$ are directional angles from incident and substrate medium, $\theta$ is penetration direction of light in the effective medium. The effective complex refractive index is denoted as $N_{\text {eff }}\left(=\sqrt{\varepsilon_{e f f}}\right)$. The optical thickness of effective medium is defined as $\delta_{e f f}=\frac{2 \pi d_{t}}{\lambda}\left(n_{e f f}-j k_{e f f}\right) \cos \theta$. The effective value of real refractive index and effective value of extinction coefficient are denoted as $n_{e f f}$ and $k_{\text {eff }}$ respectively. The total physical thickness is $d_{t}$ and the coherently illuminated spectrum for the expected design is $\lambda$. The Snell's law is defined for multi-layer case as

$$
n_{0} \sin \theta_{0}=\left(n_{e f f}-j k_{e f f}\right) \sin \theta=n_{s} \sin \theta_{\mathrm{s}}
$$

In this article, the bilayer geometry is arranged with symmetric structure $\eta_{0}=\eta_{s}$. The condition of CPA design is set when the surface admittance becomes equal to the admittance of incident medium. It can be written as

$$
Y=\frac{C}{B}=\eta_{s}
$$

The simplification of eq. $(3,5)$ yields the eigenvalue expression [37] as

$$
\tan \delta_{e f f}=\frac{-2 j\left(\frac{\eta_{e f f}}{\eta_{S}}\right)}{1+\left(\frac{\eta_{e f f}}{\eta_{s}}\right)\left(\frac{\eta_{e f f}}{\eta_{s}}\right)}
$$

This is an eigenvalue expression of CPA mode in EMA for planar thin film geometry.

The transverse resonance like state of CPA mode appears in the absorbing film which can be written from eq. (6) as

$$
\delta_{e f f}=2 \tan ^{-1}\left(\frac{\eta_{e f f}}{j \eta_{s}}\right)+2 m \pi
$$

where $\delta_{e f f}=\kappa_{e f f} d_{t}$ is transverse phase, the inverse of tangent is the reflection phase. The eq. (7) is the status of a single mode resonance and at the thinnest physical thickness of planar thin film, this mode can be obtained at zero order $m=0$. Thus admittance matching method provides the thin film design from its CPA condition and it produces the behavior of CPA mode in thin film structure as well. 


\section{3. b. Scattering Matrix Method}

The optical phenomena which are taken place in the whole configuration of Fig. 2 (a), these can be analyzed in scattering matrix method. The reflections and transmissions coefficients are represented in this formulation for the symmetric structure $\left(\eta_{0}=\eta_{s}\right)$ as

$$
\left[\begin{array}{l}
E_{\text {out } 1} \\
E_{\text {out } 2}
\end{array}\right]=\left[\begin{array}{ll}
r_{1} & t_{1} \\
t_{1} & r_{2}
\end{array}\right]\left[\begin{array}{l}
E_{\text {in } 1} \\
E_{\text {in } 2}
\end{array}\right]
$$

The input irradiances in incident and substrate medium are defined as $I_{i n 1}=\frac{1}{2} y_{0} \eta_{s}\left|E_{\text {in } 1}\right|^{2}$ and $I_{\text {in } 2}=\frac{1}{2} y_{0} \eta_{s}\left|E_{\text {in } 2}\right|^{2}$ respectively, whereas $y_{0}$ is admittance of the vacuum. Normalizing the eq. (8) which yields as

$$
\left[\begin{array}{l}
O_{1} \\
O_{2}
\end{array}\right]=\left[\begin{array}{ll}
r_{1} & t_{1} \\
t_{1} & r_{2}
\end{array}\right]\left[\sqrt{\frac{I_{i n 2}}{I_{i n 1}}} e^{-i \phi_{12}}\right]
$$

The normalized input and output amplitude fields are defined as $O_{1}=\frac{E_{\text {out } 1}}{E_{\text {in1 }}}, O_{2}=\frac{E_{\text {out } 2}}{E_{\text {in } 1}}, E_{\text {in } 1}=1$ and the relative irradiance ratio between two counter inputs is obtained by $\frac{E_{i n 2}}{E_{i n 1}}=\frac{\left|E_{i n 2}\right| e^{i \phi_{2}}}{\left|E_{i n 1}\right| e^{i \phi_{1}}}=\sqrt{\frac{I_{i n 2}}{I_{i n 1}}} e^{-i \phi_{12}}$, where $\phi_{12}$ is phase difference between two counter input waves of $E_{i n 1}$ and $E_{\text {in } 2}$.

The output system yields zero values, $O_{1}=O_{2}=0$, then the CPA conditions are obtained from eq. (9) in magnitude of reflectance, transmittance and corresponding phase relationship [37] as $\sqrt{R_{1} R_{2}}=T$ and $\phi_{t}=\left(\phi_{r 1}+\phi_{r 2}\right) / 2$ respectively. The output in the incident medium is derived from eq. (9) as

$$
=>I_{\text {out } 1}=\frac{1}{2} y_{0} \eta_{s}\left|O_{1}\right|^{2}=\frac{1}{2} y_{0} \eta_{s}\left(R_{1}+\frac{I_{\text {in } 2}}{I_{\text {in } 1}} T+2 \sqrt{\frac{I_{\text {in } 2}}{I_{\text {in } 1}}} \sqrt{R_{1} T} \cos \Delta_{1}\right)
$$

The forward phase relationship is obtained as $\Delta_{1}=\phi_{r_{1}}-\phi_{t}-\phi_{12}$

Similarly, the output in the substrate medium is derived from eq. (9) as

$$
=>I_{\text {out } 2}=\frac{1}{2} y_{0} \eta_{s}\left|O_{2}\right|^{2}=\frac{1}{2} y_{0} \eta_{s}\left[T+\frac{I_{\text {in } 2}}{I_{\text {in } 1}} R_{2}+2 \sqrt{\frac{I_{\text {in } 2}}{I_{\text {in } 1}}} \sqrt{R_{2} T} \cos \Delta_{2}\right]
$$

The backward phase relationship can be written as $\Delta_{2}=\varphi_{t}-\varphi_{r_{2}}-\varphi_{12}$.

The above derived eqs. (10-11) can be generalized and applicable for any planar and nanostructured thin films to analyze the absorption effect irrespectively in symmetric $\left(\eta_{0}=\eta_{s}\right)$ and asymmetric $\left(\eta_{0} \neq \eta_{s}\right)$ structures.

\section{Simulation Results Analysis of CPA}

\section{4. a. Analysis of Narrowband CPA}

To achieve the perfect absorption in thin film structure, it is very important in the consistency of optical constants which are produced in the films on appearance of electromagnetic wave to interact with it. The dielectric properties which belong to tunable ITO-1 as shown in the previous Fig. 1 that doesn't support the single layer CPA design. To overcome this drawback, a dielectric layer of $\mathrm{SiO}_{2}$ is used in this schematic of Fig. 3 (a). It acts as a coupling layer. It enhances the perfect resonance effect in this geometry of identical incident and substrate medium of $\mathrm{ZnSe}$. In this configuration, the sandwiching layers are designated as the layer-1 of $\mathrm{SiO}_{2}$ which is deposited on the 
substrate and then the deposited sequence of another layer-2 is as ITO- 1 . The absorbing layer lies here in the outside of dielectric layer. The incident medium is interfaced with the plasmonic layer.

The admittance matching design of narrowband CPA is carried out at the monochromatic wavelength of $1493 \mathrm{~nm}$. In this designed spectrum ITO-1 is found as the plasmonic material at the condition of optical constants as $n<k$. The design method extracts the thin film parameters such as the perfect direction and physical thickness of the corresponding layers. All of these are ensured by the admittance matching diagram of Fig. 3 (b).

It shows the loci for individual layers. The variation of trajectories occurs from the substrates for each beam to the interface between the layers in case of two counter input illuminations. The traveling path of admittance ends to contact it to the incident medium. During these phenomena, the diagrams cross in the interface and it indicate the completeness of the design as the admittance trajectory traveling from substrate to the incident medium for the multilayer thin film geometry. The admittance locus may take the forms of circular arcs or the complete circles [36]. The optical thickness of forward beam is positive. The emergent medium for this beam is substrate. It is vice-versa for backward beam. In this structure, the coordinate of forward locus (red line) starts at the modified admittance point $(-2.4487,0)$ of real axis of substrate $\left(-\eta_{s}\right)$. It traverses throughout the absorption free dielectric medium of $\mathrm{SiO}_{2}$. The coordinate of the backward locus (black line) switches at the modified admittance of another point $(2.4487,0)$ of real axis of incident medium $\left(\eta_{s}\right)$. It passes through the absorbing medium of ITO-1. The output irradiances are calculated with respect to $1493 \mathrm{~nm}$ wavelength for incident medium, substrate medium and for the effective medium approximation (EMA) as function of wavelength.

The trajectories of admittance diagrams of this bilayer geometry clarify evidently the absorption and dissipation properties of respective materials. The diagrams are obtained by using the real and imaginary coefficients of the surface admittance $(Y=\alpha+j \beta)$ in the complex plane. These values are extracted using the TMM as expressed in eq. (3) for corresponding layer. These traversing of the loci show the counter propagation directions of each beam as the time reversal symmetry $[10,11]$. The loci are interconnected at a common complex coordinate $(-2.3473,0.5911)$ of complex value of surface admittance. It occurs in the interface between the layers. The terminations of the loci occur through the common coordinate to merge it as a single locus. The merged locus ends to the real coordinates of modified admittance of each emergent medium. Using this admittance matching design of single or multilayer thin film, Fresnel coefficients and absorption condition can be calculated [36, 38].

The optical phenomena which occur in the Fig. 3(b), these can be perceived by the Fig. 3 (c). The EMA is employed to extract the optical matters over there. The optical constants of ITO-1 are obtained by the eq. (1). The same values are extrapolated by the Sellmeier equation [38] for the dielectric layer of $\mathrm{SiO}_{2}$. The effective dielectric constant $\varepsilon_{e f f}(\lambda)$ is calculated by the eq. (2). The real and imaginary parts of $\varepsilon_{e f f}(\lambda)$ are plot individually. The real part of $\operatorname{Re}\left(\varepsilon_{e f f}\right)$ which shows the development of ENZ spectrum $\left(\lambda_{\text {enz }}\right.$, eff $)$ of effective medium at $1519 \mathrm{~nm}$. It is longer than that of the illuminated monochromatic designed spectrum of $1493 \mathrm{~nm}$. Comparatively, it can be stipulated that the dielectric constants in the effective medium at the designed spectrum are shifted to the value of $N_{\text {eff }}(1493 \mathrm{~nm})=0.78201-0.583565 * 1 \mathrm{j}$. The plasmonic character is developed by the optical constants of $n_{e f f}>k_{e f f}$ as the effective medium in this spectrum. This type of partial plasmonic property is conveniently feasible to study the absorbing phenomenon at a certain infrared spectral regime of TCOs. The real and imaginary effective permittivity values are produced in this spectrum as 0.270992 and 0.912708 respectively. These values are less than unity. It resembles robustly with the production potentiality of perfect absorption resonance effect $(12,29)$. The narrowband CPA designed is found in this specific condition of EMA.

Thus dielectric layer ensures the optimum condition of optical geometry with enhancement of narrowband resonance effect. The real part of $\operatorname{Re}\left(\varepsilon_{e f f}\right)$ shows the location of plasmonic character which clarifies the spectrum regime of unbound electrons in EMA. The sharply falling line of real value of effective permittivity which produces higher value of negative slope in comparison with the tunable ITO-1 in Fig. 1. This phenomenon makes the distinction of effective permittivity in EMA with the intrinsic permittivity for solely single layer tunable ITO- 1 . Thus CPA is enhanced with sensitiveness to these values of real and imaginary parts of effective dielectric constants $\left(\varepsilon_{e f f, r e}, \varepsilon_{e f f, i m g}\right)$. 

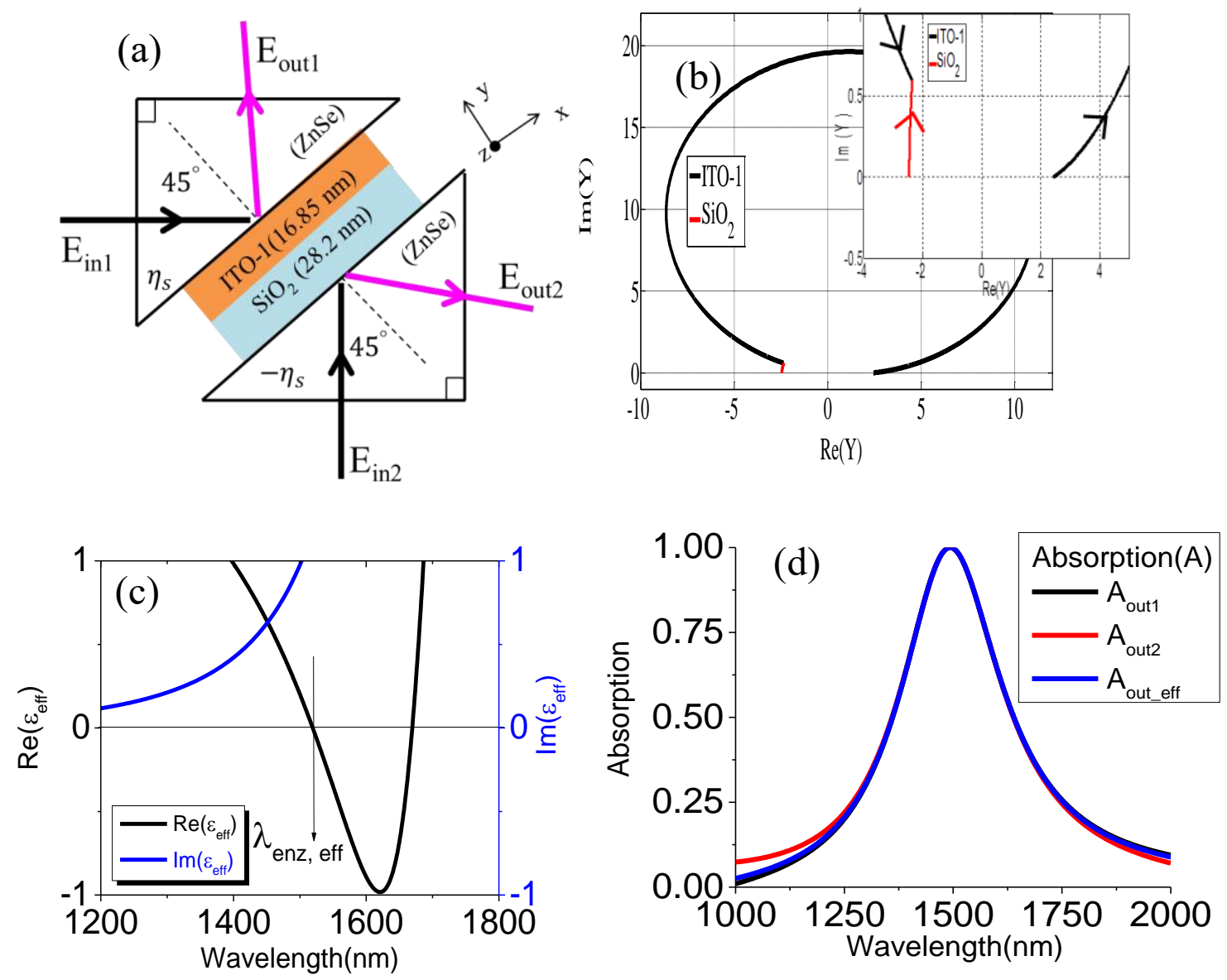

Figure 3: (a) Schematic diagram of narrowband CPA in symmetric structure of bilayer thin film with refractive index of $\mathrm{ZnSe}$ is as $N_{\mathrm{ZnSe}}(1493 \mathrm{~nm})=2.44874$, (b) Admittance matching design for the geometry of (a) at 1493 $\mathrm{nm}$ wavelength in which the complex refractive index of tunable ITO-1 is as $N_{\text {ITO-1 }}(1493 \mathrm{~nm})=0.430766-$ $0.431178 * 1 \mathrm{j}$ and the refractive index of dielectric layer of $\mathrm{SiO}_{2}$ is used as $N_{\text {SiO2 }}(1493 \mathrm{~nm})=1.4447$, (c) The complex value of effective dielectric constant of schematic (a) is calculated as, $\varepsilon_{e f f}=\left(n_{e f f}-j k_{e f f}\right)^{2}$ with development of the effective ENZ position $\left(\lambda_{\text {enz }}\right.$ eff $)$ at $N_{\text {eff }}(1519 \mathrm{~nm})=0.767014-0.764171 * 1 \mathrm{j}$ and $(\mathrm{d})$ The output absorptions are calculated with respect to $1493 \mathrm{~nm}$ wavelength for incident medium, substrate medium and for the effective medium approximation (EMA) as function of wavelength.

Now the output absorption spectra $\left(A_{\text {out }}, A_{\text {out } 2}, A_{\text {out_eff }}\right)$ from the designed structure can be calculated by the eqs. $(10,11)$ as the Fig. 3 (d) is shown. Transfer matrix method (TMM) software [40] is used to calculate the magnitudes, corresponding phases of reflectance and transmittance in the respective emergent medium. The two counter input phase difference $\phi_{12}$ and the irradiance ratio of $\frac{I_{\text {in } 2}}{I_{\text {in } 1}}$ are also obtained by TMM. The calculated absorption is more than $99 \%$ in each emergent medium at the designed monochromatic spectrum. Beyond the phase and irradiance matching, the scattered irradiance enhances and reduces the absorption gradually. This is the indication of a modulator application of counter-inputs CPA. The absorptions in lower and higher ranges beyond the CPA wavelength are very small in forward and backward inputs because of nearly transparency structure effect in those spectral regimes. This CPA mode appears due to the radiative resonance effect as it is explained in the Fig. 3(c). The resonant spectrum position of effective permittivity of real part shows it evidently. The developing CPA mode doesn't cross the ENZ spectrum [12] of effective medium. The absorption spectra in EMA method also show the well matching with all the output characteristics as it is expected.

Two alternative methods are applied here to verify the CPA conditions in scattering matrix separately. The Fig. 4 (a) represents graphically at variable wavelength, the product of magnitude of reflectance in each medium 
(black curve) and transmittance (blue curve) $\left(T=\sqrt{R_{1} R_{2}}\right)$. These spectra show the single intersection coordinate which is corresponding to the CPA monochromatic designed wavelength. This resonance coupling produces the destructive interference effect as CPA at coherent wavelength of phase matching condition. Beyond the resonance wavelength constructive interference enhances as the transmittance spectrum show which is a single dip. The opposite phenomena occur in case of spectrum of reflectance especially around the resonance wavelength.
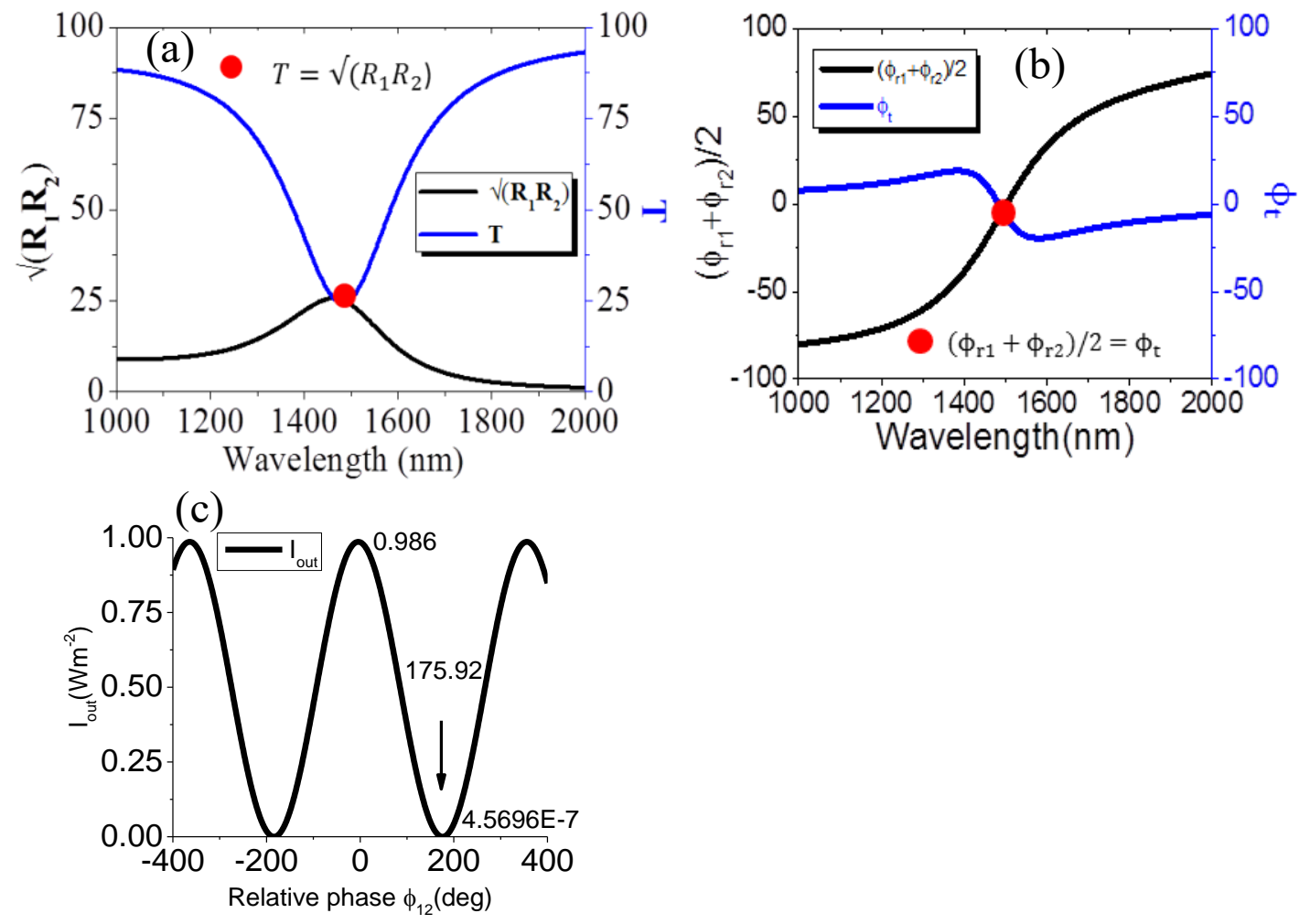

Figure 4: (a) The satisfaction of narrowband CPA condition at the magnitudes of transmittance and reflectance relationship, (b) Similarly, the satisfaction of narrowband CPA condition at phase relationship of reflection and transmission coefficients and (c) The normalized irradiances of $I_{\text {out }}$ is calculated as the function of relative phase shift $\left(\varphi_{12}\right)$ between two counter inputs.

The Fig. 4 (b) shows the phase relationship of reflection and transmission coefficients $\left(\phi_{t}=\left(\phi_{r 1}+\phi_{r 2}\right) / 2\right)$. The total reflection phase of incident and substrate medium slightly falls as wavelength increases which is noticeable around the CPA wavelength. Expectedly, at the same spectral position, opposite phenomenon occurs in case of transmission phase. The intersection point of both phases is located in this regime which is also corresponding to the CPA. In these ways the phase matching conditions are satisfied separately.

The normalized output irradiances are also plot in Fig. 4 (c) as function of relative input phase in CPA wavelength. It shows that beyond the relative phase of $175.92^{\circ}$ in degree of CPA mode, the irradiances increase as the effect of constructive interference between scattered amplitudes in each output medium. The maximum value tends to the transparency state of 0.986 . The minimum value approaches to zero approximately as $4.57 \times 10^{-7}$. Thus the sensitivity of relative phase between two counter input beams is obvious. This property is suitable to optical filter or optical switching and modulation applications.

\section{4. b. Analysis of broadband CPA}

It is very unlikely to achieve broadband perfect absorption in a single layer of any plasmonic material. On the other hand, a single dielectric layer is unable to produce broadband CPA with a plasmonic layer. Thus two tunable ITOs are deposited over substrate by turn as the Fig 5 (a) is presented to overcome this problem. Controlling the carrier concentration to the optimum level is very important task to find these tunable materials [4, 33-34]. Two 
tunable ITOs broadly enhance the perfection of resonance effect in this geometry. Thus the used layers are designated as the layer-1 of ITO-2 which is deposited on the substrate and then the deposited sequence of another layer-2 is as ITO-1.
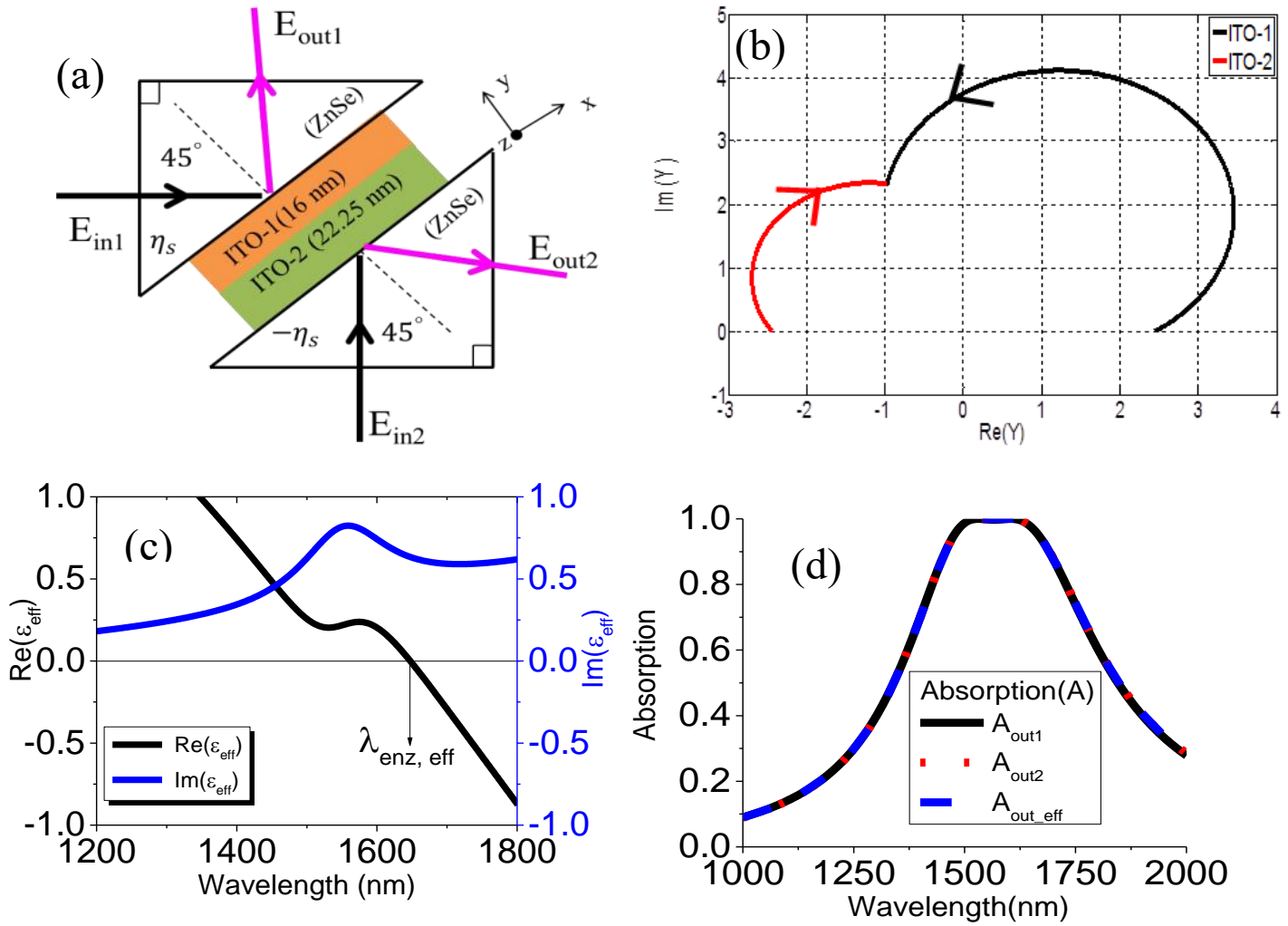

Figure 5: (a) Schematic diagram of broadband CPA in symmetric structure of bilayer thin film with refractive index of $\mathrm{ZnSe}$ is as $N_{\mathrm{ZnSe}}(1530 \mathrm{~nm})=2.44855$, (b) Admittance matching design for the structure of (a) at $1530 \mathrm{~nm}$ wavelength in which the complex refractive indices of tunable ITO-1 and ITO-2 are calculated as $N_{\text {ITO- } 1}(1530 \mathrm{~nm})=0.35361-0.56503 * 1 \mathrm{j}$ and $N_{\text {ITO-2 }}(1530 \mathrm{~nm})=0.81434-0.19628 * 1 \mathrm{~J}$ respectively, $(\mathrm{c})$ The complex value of effective dielectric constant is calculated for the schematic of (a) as, $\varepsilon_{e f f}=\left(n_{e f f}-j k_{e f f}\right)^{2}$ with development of the effective ENZ position at $N_{e f f}(1647 \mathrm{~nm})=0.564335-0.56203 * 1 \mathrm{j}$ and (d) The broadband output absorptions are calculated at $(1498-1641 \mathrm{~nm})$ range wavelengths with respect to reference wavelength $1530 \mathrm{~nm}$ for incident medium, substrate medium and for the effective medium approximation (EMA) as function of wavelength.

The broadband CPA thin film structure is designed by the Fig. 5 (b). The reference spectrum is always arbitrary and helpful to extract the optimum optical constants for a bilayer broadband design. In our approaches, it is found that even at the same bilayer thin film structure, the broadband CPA design is difficult to obtain at a certain spectrum. The obtainment of CPA thin film design is little easy, if the designed spectrum ensures the plasmonic character as $n<k$ for one film and plasmonic character with condition of $n>k$ for other film in aspect of the fractional optical constant less than unity of ENZ materials. Finally, the optimum optical constants were found for the broadband CPA design of monochromatic wavelength at $1530 \mathrm{~nm}$. In this structure, the coordinate of forward locus (red line) starts at the modified admittance point $(-2.4487,0)$ of real axis of substrate $\left(-\eta_{s}\right)$. It traverses clockwise direction throughout the layer-1 of ITO-2. The coordinate of the backward locus (black line) switches at the modified admittance of another point $(2.4487,0)$ of real axis of incident medium $\left(\eta_{s}\right)$. It passes anticlockwise direction throughout the layer- 2 of ITO- 1 . The loci are interconnected at a common complex coordinate $(-0.9785$, 2.3086) of complex value of surface admittance. It occurs in the interface between the layers. The terminations of the loci occur through the common coordinate to merge it as a single locus. The merged locus ends to the real coordinates of modified admittance of each emergent medium. 
The optical phenomena which occur in the Fig. 5 (b), these can be perceived by the Fig. 5 (c) in EMA method. The optical constants of ITO-1 \& 2 are obtained by the eq. (1). The real and imaginary parts of $\varepsilon_{e f f}(\lambda)$ are plot individually. The real part of $\operatorname{Re}\left(\varepsilon_{e f f}\right)$ which shows the development of ENZ spectrum $\left(\lambda_{\text {enz, eff }}\right)$ of effective medium at $1647 \mathrm{~nm}$. It is longer than that of the illuminated monochromatic designed spectrum. The comparative values of the dielectric constants at designed spectrum are shifted to $N_{e f f}(1530 \mathrm{~nm})=0.704417-0.541234 *$ $1 j$. It ensures the plasmonic property as $n_{e f f}>k_{e f f}$ at the effective medium. The radiative mode developing spectral regimes must be shorter than that of ENZ spectrum of effective medium. The real and imaginary effective permittivity values should be produced as less than unity. It is an important ENZ property which is noticeable in the spectral regimes of $1352 \mathrm{~nm}-1647 \mathrm{~nm}$. Thus broadband CPA designed is found in this specific condition of EMA method [29]. The real part of $\operatorname{Re}\left(\varepsilon_{e f f}\right)$ shows the location of plasmonic character which clarifies the spectrum regime of unbound electrons in EMA method.

The eqs. $(10,11)$ are also used to calculate the output absorption spectra $\left(A_{\text {out }}, A_{\text {out } 2}, A_{\text {out_eff }}\right)$ by TMM software [40] in the corresponding emergent medium as the Fig. 5 (d) is shown. The calculations show that $99 \% \geq$ absorption appears at the broadband spectral regimes of $1498 \mathrm{~nm}-1641 \mathrm{~nm}$. This broadband CPA mode appears due to the radiative resonance effect as it is explained in the Fig. 5(c). The broadband resonant spectral regimes of effective permittivity of real part show it evidently. The developing broadband CPA mode spectral regimes are less than that of the ENZ spectrum of the effective medium [29]. The broadband CPA output result is also calculated to verify these results at incident medium in EMA method. The all regimes of spectra show the well matching with all the output characteristics as it is expected.

Previous two alternative methods are also applied here to verify the broadband CPA conditions in scattering matrix separately. The Fig. 6 (a) represents graphically at variable wavelength, the product of magnitude of reflectance in each medium (black curve) and transmittance (blue curve) $\left(T=\sqrt{R_{1} R_{2}}\right)$. The spectral lines are nearly tends to be flat in the broadband CPA spectral regimes. These spectral lines show the multi-intersection coordinates which are corresponding to the $1426 \mathrm{~nm}, 1530 \mathrm{~nm}, 1605 \mathrm{~nm}$ and $1764 \mathrm{~nm}$ spectral positions respectively. The middle two spectrum lies in the broadband CPA spectral range of $1498 \mathrm{~nm}-1641 \mathrm{~nm}$. These multi-resonance coupling enhances the destructive interference effect around these to broadband CPA at coherent spectral regime of phase matching condition. Beyond the resonant spectral regimes constructive interference enhances to the transparency state of incoherency effect. The opposite phenomena occur in case of reflectance especially around the resonant spectral regimes.

The Fig. 6 (b) shows the phase relationship of reflection and transmission coefficients $\left(\phi_{t}=\left(\phi_{r 1}+\phi_{r 2}\right) / 2\right)$. It shows that reflection coefficients phase tends to rise and then it becomes flat, uniform and then again it begins to rise. The transmission coefficient exhibits the opposite phenomena of reflection coefficient phase throughout whole spectral regimes. There is a calculated broadband $1517 \mathrm{~nm}-1617 \mathrm{~nm}$ spectral range of phase matching condition as flat line and nearly uniform in magnitude. The intersection points of both phases are located in these regimes. The broadband CPA of $1498 \mathrm{~nm}-1641 \mathrm{~nm}$ spectral range is little beyond this range as mentioned previous. This indicates that there is a range of maximal phase difference to cause the destructive interference in this type of multilayer design at coherent condition. In these ways the phase matching conditions are satisfied separately.

The Figure 6 (c) represents the filled contour plot as function of relative phase shift $\varphi_{12}$ in degree at corresponding wavelength. The color bar shows the distribution of the state of output port irradiance in the incident medium. In this plot the increment of $\varphi_{12}$ is 1 and using the each single value of relative phase, the output port irradiances are calculated at each wavelength at the range of $1000 \mathrm{~nm}-2000 \mathrm{~nm}$. These calculations are continuing in the range of relative phase from 0-360 degree for this range of wavelength. The relative phase difference $\varphi_{12}$ between two counter input beams is found as $178.759^{\circ}$ in this plot at the designed wavelength $1530 \mathrm{~nm}$. If we look at the $179^{0}$ of $\varphi_{12}$ in the scale then the corresponding output irradiance is observable less than $1 \%$ in the color bar. This minimal value exists in the broadband CPA range of $1503 \mathrm{~nm}-1635 \mathrm{~nm}$. Thus the leakage of irradiance at broadband time-reversal CPA is nearly zero. On the other hand, output port irradiance is high beyond the phase matching regime in lower and upper scales. In these ways, broadband calculations show the consistent behavior in each method of this analysis. This design can be applicable for broadband modulator and energy harvesting devices.

In brief discussion, EMA method is used here to extract the effective dielectric constant of the multilayer planar thin film for characterization of CPA modes. Admittance matching method can be utilized to make a convenient design of thin film before fabrication. It is also found that the ENZ properties can be harvested at the optimum condition of fractional optical constants such as $0<n, k<1$ at the corresponding spectral regimes of the material. Using this fractional condition, the consecutive combination of plasmonic ENZ layer with spectral regime 
of $n<k$ and the additional plasmonic ENZ layer with spectral regime of $n>k$ is convenient for development of broadband CPA. The complete dielectric layer $(k=0)$ always plays a role of enhancement of narrowband CPA effect. The relative phase controlling effect between the counter input beams at the resonance regimes in these developed planar thin film designs may be functional for modulator, optical switching and infrared sensor. These absorbing bilayer optical thin films have also energy conversion effect in integrated optical devices.

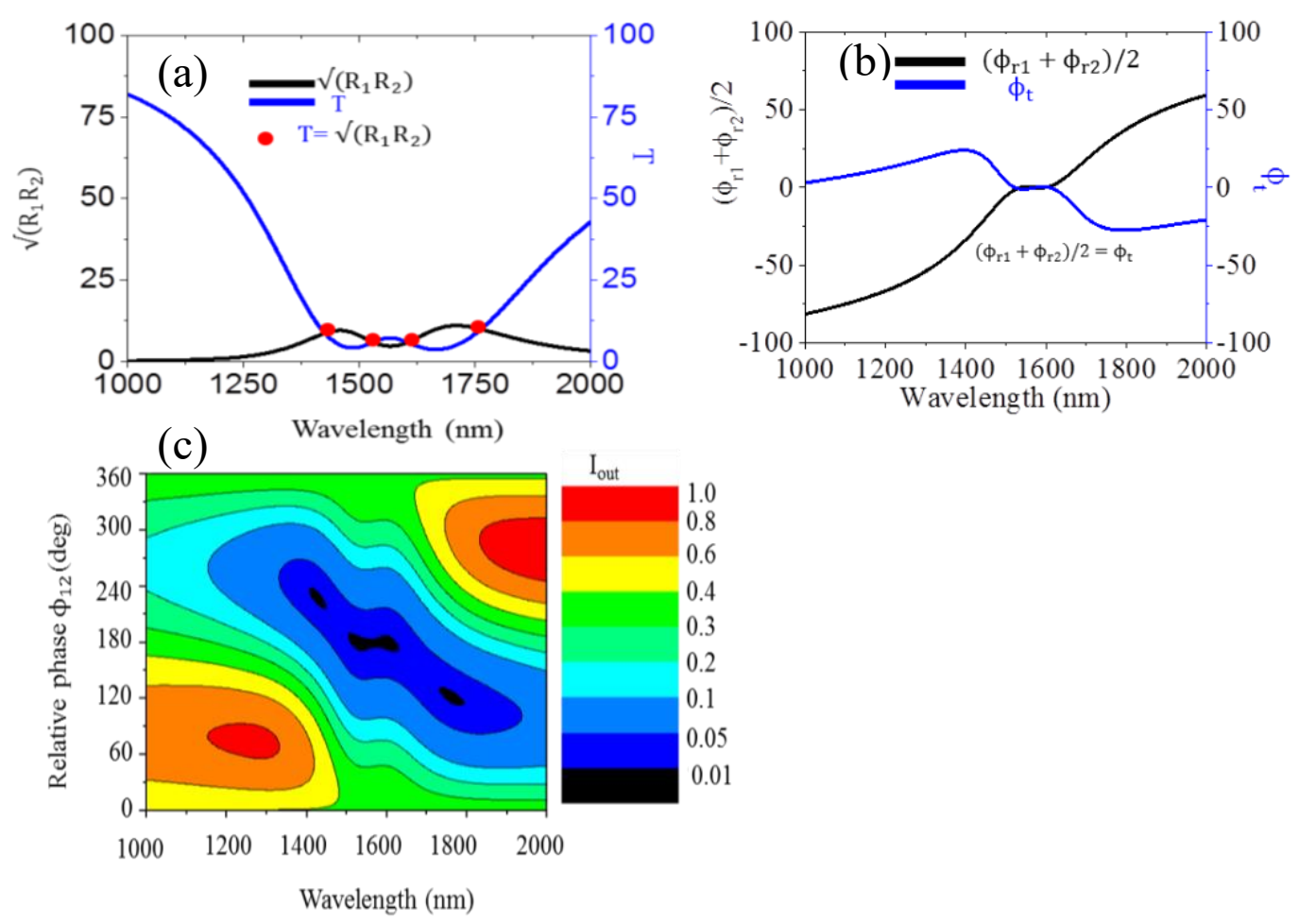

Figure 6: (a) The satisfaction of broadband CPA condition at the magnitudes of transmittance and reflectance relationship, (b) Similarly, the satisfaction of broadband CPA condition at phase relationship of reflection and transmission coefficients and (c) The normalized irradiances of broadband $I_{\text {out }}$ is calculated as the function of relative phase shift $\left(\varphi_{12}\right)$ between two counter inputs.

\section{Conclusion}

In this study, it is found that at the designed spectrum in the optimum condition of fractional optical constants less than unity of ENZ material, the consecutive combination of plasmonic layer at $n<k$ and additional dielectric $(k=0)$ or plasmonic layer at $n>k$ multilayer enables to obtain the CPA conditions conveniently in admittance matching method. This geometrical configuration agrees with the enhancement of the plasmons resonant effect. The broadband and narrowband CPAs wavelength spectral regimes are shorter than that of ENZ spectrum of the effective medium. Thus admittance matching designed structures produce here radiative resonant effect like CPA modes characteristically. It is attributable to the plasmons resonant effect in the plasmonic layer. The discussing method is superior over one channel CPA and MMs on phase controlled phenomenon. The broadband CPA paves the way of energy transforming mechanism likely in photovoltaic cell and the advanced stealth technological system.

\section{Acknowledgements}

This work is supported by the research project of Jashore University of Science and Technology, Jashore-7408, Bangladesh. 